\title{
Hepatitis B Virus Infection in the General Population of Iran: An Updated Systematic Review and Meta-Analysis
}

\author{
Mostafa Salehi-Vaziri, ${ }^{1}$ Farzin Sadeghi, ${ }^{2}$ Amir Almasi Hashiani, ${ }^{3}$ Mohammad Gholami Fesharaki, ${ }^{4}$ and \\ Seyed Moayed Alavian ${ }^{5, *}$ \\ ${ }^{1}$ Department of Arboviruses and Viral Hemorrhagic Fevers, Pasteur Institute of Iran, Tehran, IR Iran \\ ${ }^{2}$ Cellular and Molecular Biology Research Center, Health Research Institute, Babol University of Medical Sciences, Babol, IR Iran \\ ${ }^{3}$ Medical Ethics and Law Research Center, Shahid Beheshti University of Medical Sciences, Tehran, IR Iran \\ ${ }^{4}$ Department of Biostatistics, Faculty of Medical Sciences, Tarbiat Modares University, Tehran, IR Iran \\ ${ }_{5}^{5}$ Baqiyatallah Research Center for Gastroenterology and Liver Diseases, Baqiyatallah University of Medical Sciences Tehran, IR Iran \\ "Corresponding author: Seyed Moayed Alavian, Baqiyatallah Research Center for Gastroenterology and Liver Diseases, Baqiyatallah University of Medical Sciences Tehran, IR \\ Iran. Tel/Fax: +98-2188945186, E-mail: alavian@thc.ir
}

Received 2015 December 17; Revised 2016 February 02; Accepted 2016 February 07.

\begin{abstract}
Context: The hepatitis B virus (HBV) is a major global public health problem, affecting more than 2 billion people worldwide. Accurate and updated data on HBV prevalence is important for further planning to control the infection. The aim of this study was to update the prevalence estimate of HBV infection in the general population of Iran.

Evidence Acquisition: A systematic review was done for data on the prevalence of HBV infection in the general Iranian population published between Jan. 1, 1990, and Jan. 1, 2016, in both international and national databases, including PubMed, Scopus, Web of Science, Scientific Information Database, IranMedex, and Magiran. All papers with clearly described time and location of the study, proper sampling strategies, and proper analysis methods were included in the present study. Data were extracted by two independent reviewers. Prevalence of HBV infection with a 95\% confidence interval (CI) was calculated using Stata software, version 13.

Results: The polled estimated prevalence of HBV infection in the general population of Iran was 2.2\% (95\% CI:1.9\% - 2.6\%). The highest prevalence of HBV infection (8.9\%, 95\% CI: 7.6\% - 10.2\%) was reported from Golestan province, and the lowest prevalence (0.7\%, 95\% CI: $0.4 \%-1.1 \%$ ) was seen in Kermanshah province. The prevalence of HBV infection was estimated at 3\% (95\% CI: $2.2 \%-3.8 \%$ ) for Iranian males and 1.7\% (95\% CI: 1.2\% - 2.3\%) for Iranian females. The prevalence of HBV infection in the general population of Iran was $2.9 \%$ ( $95 \%$ CI: $2.5 \%-3.4 \%$ ) before 2010 and $1.3 \%$ (95\% CI: 0.9\% - 1.7\%) after 2010.

Conclusions: In total, Iran was classified within the low-intermediate HBV prevalence areas ( $2 \%$ - $4 \%$, while according to recent data (after 2010), Iran was classified within the low HBV prevalence areas $(<2 \%)$, indicating that preventive measures conducted in Iran have been effective.
\end{abstract}

Keywords: Hepatitis B Virus, Iran, Meta-Analysis, Prevalence

\section{Context}

The Hepatitis B virus (HBV) is a major global public health problem affecting more than 2 billion people throughout the world (1). Despite the availability of a highly effective vaccine against HBV, 350 million people are estimated to be HBV chronic carriers worldwide $(2,3)$. People chronically infected with HBV are at increased risk of developing HBV-related liver diseases, including hepatic cirrhosis and hepatocellular carcinoma $(4,5)$. The estimated worldwide mortality is more than 780,000 deaths annually (6).

The frequency of HBV infection varies throughout the world $(3,7)$. There are high-prevalence areas where $>8 \%$ of the population is positive for the hepatitis B surface antigen (HbsAg); intermediate-prevalence areas in which $2 \%$ -
$8 \%$ of the population is HBsAg positive and low-prevalence areas in which $<2 \%$ of the population is HBsAg positive ( 7 , $8)$. However, because there are regions very close to lowprevalence areas and also close to high-prevalence areas, it has been suggested that splitting the intermediate category into low-intermediate-prevalence (2\% - 4.99\%) and high-intermediate-prevalence areas ( $5 \%-7.99 \%)$ could better reflect regional differences and their importance (3, 9). According to a recent study on the global epidemiology of HBV infection, Iran is classified within the lowintermediate-prevalence areas (3).

The overall prevalence of HBV infection in Iran was 1.7\% in the 1990 s $(10,11)$. However, other regional studies in various provinces of Iran showed HBV prevalence more than $1.7 \%$ in the last decade (12-14). 
To decrease HBV carriers in the community, using vaccination is one of the most beneficial strategies in reducing the frequency of HBV infection (15). Accurate and updated knowledge of the prevalence of HBV infection is necessary to assess the impact of prevention and control measurements, including vaccination programs, and also for bringing up to date the estimates of disease burden (9). However, only two systematic reviews have estimated the prevalence of $\mathrm{HBV}$ infection in the general population of Iran: the study by Alavian et al. (16), covering studies from seven provinces from 2001 to 2007, and the study by Poorolajal et al. (17), covering studies from three provinces from 1989 to 2005. Therefore, there are no up-to-date national systematic reviews reporting HBV seroprevalence in the general population of Iran.

\section{Objectives}

The present study was conducted to make an updated estimate of the prevalence of HBV infection in Iran by a systematic review of peer-reviewed publications representing HBV seroprevalence in the general population of different provinces for which epidemiological information was available.

\section{Methods}

\subsection{Search Strategy and Study Selection}

The present systematic review and meta-analysis was performed according to the criteria of the PRISMA guidelines (18). A systematic review on literature published between January 1, 1990, and January 1, 2016, was performed to find scientific papers reporting the prevalence of HBV infection (by detection of the HBsAg in samples) in the general Iranian population. To identify articles, international databases (PubMed, EMbase, Scopus, Web of Science, CABI, CINAHL, DOAJ, Index Medicus for Eastern Mediterranean Region-IMEMR, EMROMedex, High-Wire Press, and DARE ) and national databases (scientific information database (SID), IranMedex, and Magiran) were searched for MeSH terms "Hepatitis B," "HBV," and "Iran" and their Persian equivalents in titles and/or abstracts.

All publications were recorded and categorized by the use of Endnote X3. The search results were subjected systematically to title and abstract screening. Then, qualified studies were obtained for full text screening. All potentially relevant publications were reviewed independently by two reviewers (MS-V and FS) for the eligibility criteria. Discrepancy between reviewers was resolved by consensus with an expert (SMA). In this study, blinding and task separation also were applied for study selection.
All English or Persian observational (descriptive/analytical cross-sectional studies/surveys) studies on seroprevalence of $\mathrm{HBV}$ with clearly described time and location of the study, proper sampling strategies (findings could be generalized to general population), reliable laboratory techniques and proper analysis methods were considered to include in the present study. The exclusion criteria were (1) studies conducted as case reports, surveillance reports, letters or correspondence, and systematic reviews or meta-analyses; (2) overlapping studies; (3) studies performed exclusively on high-risk population groups; and (4) studies with non-serum-based HBV detection assay, such as saliva testing.

\subsection{Data Extraction and Quality Assessment}

After review of the full texts, the following data were extracted from each study: study characteristics (first author's name, date of study, period of sample collection, and province or city of the study), participant characteristics (gender, age, population group, and sample size), HBV prevalence (HbsAg seroprevalence in all cases and HbsAg seroprevalence according to gender), and HBsAg detection methods. The quality of the included study was assessed using a modified STROBE checklist (19).

\subsection{Statistical Analysis}

Statistical analysis was done by Stata software, version 13 (Stata Corp, College Station, TX, USA). The prevalence of HBV was polled by the "metan" command and showed by a forest plot for the general population and also was based on both genders. The random effect model was used for meta-analysis tests. The forest plot as a graphic manner was used to show the effect size of all the studies with their confidence intervals and the results of the metaanalysis (20). Statistical heterogeneity was explored using the I-square at the 5\% significance level. Meta-regression analysis was performed for determination of responsible factors for heterogeneity using the "metareg" command. Publication bias was assessed by Begg's and Egger's tests $(21,22)$. To correct publication bias, the Trim and Filled method was used by the "metatrim" command. Cumulative meta-analysis based on the time was performed using the "metacum" command. Sensitivity analysis was performed using the "metainf" command. The prevalence of HBV was showed in geographic maps using Esri ArcMap GIS version 10.1.

\section{Results}

\subsection{Studies}

Following the removal of duplicates and primary screening, 40 publications were reviewed in full, of which 
20 met the eligibility criteria and were included in the meta-analysis (Figure 1). Among 20 included studies, two studies $(10,11)$ were national, one study (year 2006) was conducted in Golestan, Hormozgan, and Tehran provinces (23), and 17 studies were regional (Table 1). Of the regional publications, two were from Golestan province, covering the years 2003 - $2006(14,24)$; two were from Hamadan province, covering the years 1998 - $2003(12,25)$; three were from Razavi Khorasan province, covering the years 1998 2011 (13, 26, 27); and two were from Sistan and Baluchestan province, covering the years 2008 - $2010(28,29)$. Other studies were from Charmahal and Bakhtiari (years 2012 2013) (30), East Azarbaijan (year 2001) (31), Isfahan (year 2006) (32), Kermanshah (year 2010) (33), Kordestan (year 2010) (34), Mazandaran (years 2008 - 2011) (35), Qom (year 2010) (36), and south Iran (years 2008 - 2009) (37).

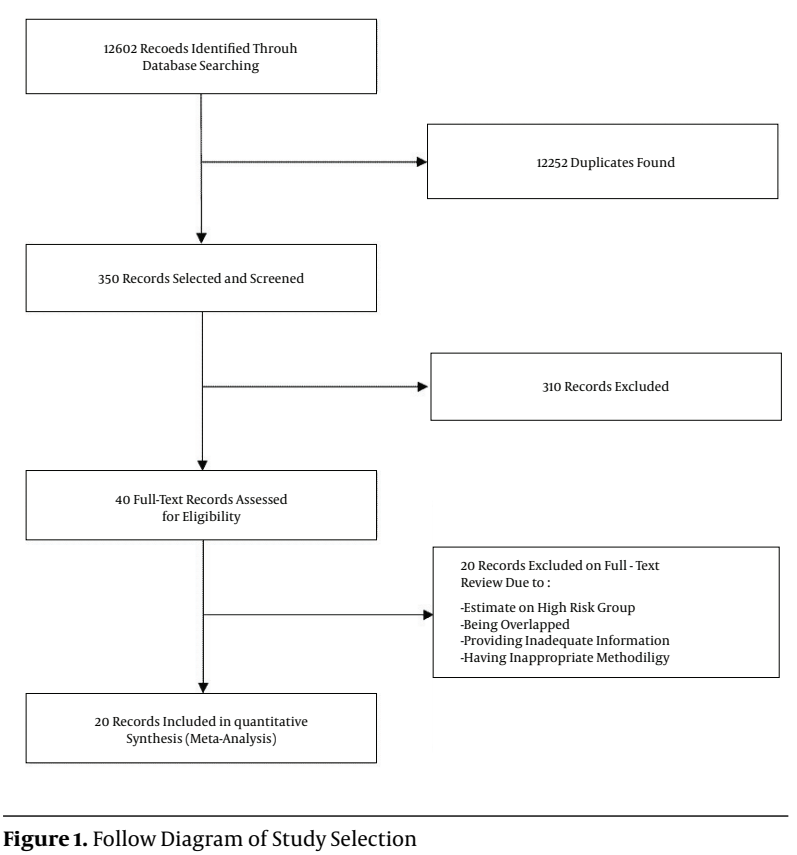

All included papers were cross-sectional studies. The sample size of the studies was between 381 and 46,631 and the age of study subjects was 1 - 100. Overall, most study subjects were females (Table 1 ). In all included studies, reliable commercial HbsAg ELISA kits were employed.

\subsection{Prevalence of $H B V$ Infection}

The polled estimated prevalence of HBV infection in the general population of Iran from 1990 to 2016 was 2.2 \% (95\% CI: 1.9\% - 2.6\%) (Figure 2). The highest prevalence of HBV infection (8.9\%, 95\% CI: 7.6\% - 10.2\%) was reported from Golestan province (14), and the lowest prevalence $(0.7 \%$, 95\% CI: 0.4\% - 1.1\%) was seen in Kermanshah province (33).

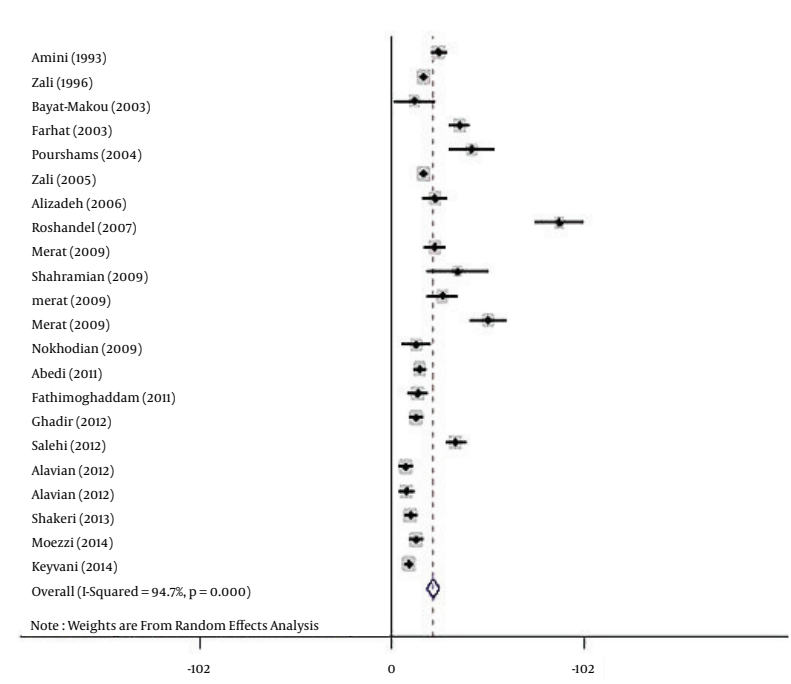

Figure 2. Forest Plot Showing Prevalence of HBV Infection in the General Iranian Population

Regarding HBV infection according to gender, the prevalence of $\mathrm{HBV}$ infection was estimated at 3\% (95\% CI: $2.2 \%-3.8 \%$ ) and $1.7 \%$ (95\% CI: $1.2 \%-2.3 \%$ ) for Iranian males (Figure 3) and females (Figure 4), respectively.

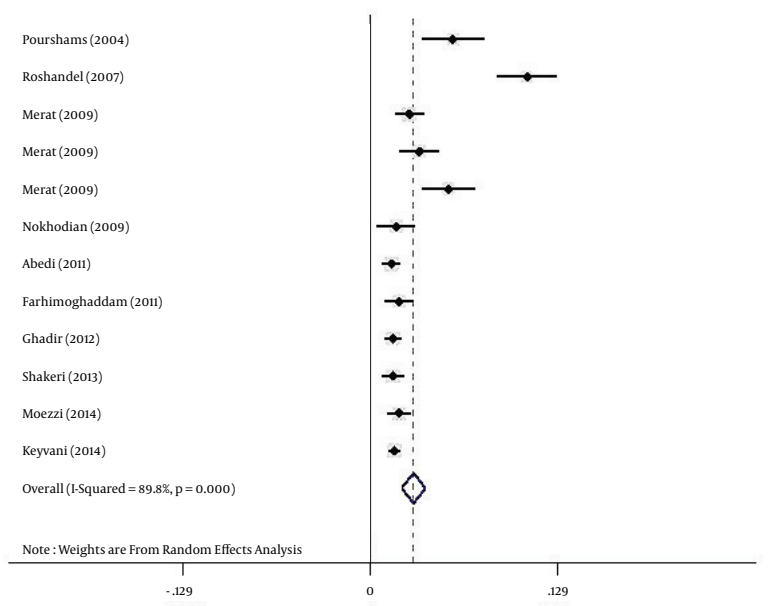

Figure 3. Forest Plot Showing HBV Prevalence in the General Male Iranian Population

The results of the heterogeneity test indicated that the studies were significantly heterogeneous $(\mathrm{P}=0.001)$, so the random model was used for polling the data. To determine responsible factors for heterogeneity, a meta-regression 
Table 1. Estimations of the Prevalence of HBV Infection in the General Population of Iran and its Provinces

\begin{tabular}{|c|c|c|c|c|c|c|}
\hline Province & $\begin{array}{c}\text { Study's first author (year of } \\
\text { publication) }\end{array}$ & Year of Study & Total No. & Male No. & $\begin{array}{l}\text { HBV Prevalence estimates \% } \\
\text { (95\% CI) }\end{array}$ & Reference \\
\hline Charmahal and Bakhtiari & Moezzi (2014) & $2012-2013$ & 3000 & 1111 & $1.3(0.9-1.7)$ & (30) \\
\hline East Azarbaijan & Bayat-Makou (2003) & 2000 & 381 & ND & $1.2(0.1-2.3)$ & (31) \\
\hline \multicolumn{7}{|l|}{ Golestan } \\
\hline Study 1 & Pourshams (2004) & 2003 & 1035 & 438 & $4.3(3-5.5)$ & $(24)$ \\
\hline Study 2 & Roshandel (2007) & $2004-2005$ & 1850 & 877 & $8.9(7.6-10.2)$ & $(14)$ \\
\hline Study 3 & Merat (2009) ${ }^{\mathrm{a}}$ & 2006 & 1896 & 607 & $5.1(4.1-6.1)$ & (23) \\
\hline Total & & & & & $6.1(3.5-8.7)$ & \\
\hline \multicolumn{7}{|l|}{ Hamadan } \\
\hline Study 1 & Amini (1993) & 1989 & 4930 & 1649 & $2.5(2.1-2.9)$ & $(25)$ \\
\hline Study 2 & Alizadeh (2006) & 2003 & 1824 & 1025 & $2.3(1.6-3)$ & $(12)$ \\
\hline Total & & & & & $2.4(2.1-2.8)$ & \\
\hline Hormozgan & Merat (2009) & 2006 & 1455 & 658 & $2.7(1.9-3.5)$ & (23) \\
\hline Isfahan & Nokhodian (2009) & 2006 & 816 & 388 & $1.3(0.5-2.1)$ & (32) \\
\hline Kermanshah & Alavian (2012) & 2010 & 1979 & 990 & $0.7(0.4-1.1)$ & (33) \\
\hline Kordestan & Alavian (2012) & 2010 & 1613 & 548 & $0.8(0.4-1.2)$ & $(34)$ \\
\hline Mazandaran & Keyvani (2014) & $2008-2011$ & 6146 & 3472 & $0.9(0.7-1.2)$ & (35) \\
\hline Qom & Ghadir (2012) & 2010 & 3666 & 1709 & $1.3(0.9-1.7)$ & (36) \\
\hline \multicolumn{7}{|l|}{ Razavi Khorasan } \\
\hline Study 1 & Farhat (2003) & 1998 & 4528 & ND & $3.6(3.1-4.1)$ & $(13)$ \\
\hline Study 2 & Fathimoghaddam (2011) & 2009 & 1652 & 794 & $1.4(0.8-2)$ & (26) \\
\hline Study 3 & Shakeri (2013) & $2010-2011$ & 3198 & 1021 & $1(0.7-1.3)$ & $(27)$ \\
\hline Total & & & & & $2(0.4-3.6)$ & \\
\hline \multicolumn{7}{|l|}{ Sistan and Baluchestan } \\
\hline Study 1 & Shahramian (2009) & 2008 & 458 & 221 & $3.5(1.8-5.2)$ & $(28)$ \\
\hline Study 2 & Salehi (2012) & 2010 & 3989 & 1835 & $3.4(2.8-4)$ & (29) \\
\hline Total & & & & & $3.4(2.9-3.9)$ & \\
\hline Southern Iran & Abedi (2011) & $2008-2009$ & 4087 & 1292 & $1.5(1.1-1.9)$ & $(37)$ \\
\hline Tehran & Merat (2009) $)^{a}$ & 2006 & 2327 & 968 & $2.3(1.7-2.9)$ & (23) \\
\hline \multicolumn{7}{|l|}{ National } \\
\hline Study 1 & Zali (1996) & 1991 & 39841 & ND & $1.7(1.6-1.8)$ & $(10)$ \\
\hline Study 2 & Zali (2005) & 1999 & 46631 & ND & $1.7(1.6-1.8)$ & (11) \\
\hline Total & & & & & $1.7(1.6-1.8)$ & \\
\hline Overall & & & & & $2.2(1.9-2.6)$ & \\
\hline
\end{tabular}

Abbreviation: ND, not determine.

${ }^{\mathrm{a}}$ This study was conducted in Golestan, Hormozgan, and Tehran provinces.

analysis was carried out. This analysis revealed that the time is responsible for heterogeneity. Therefore, a subgroup analysis and a cumulative meta-analysis based on the time were performed. In the subgroup analysis, the studies were divided into two different time periods, including before and after 2010. After subgroup analysis, heterogeneity was removed. The polled estimated prevalence of HBV infection in the general population of Iran 


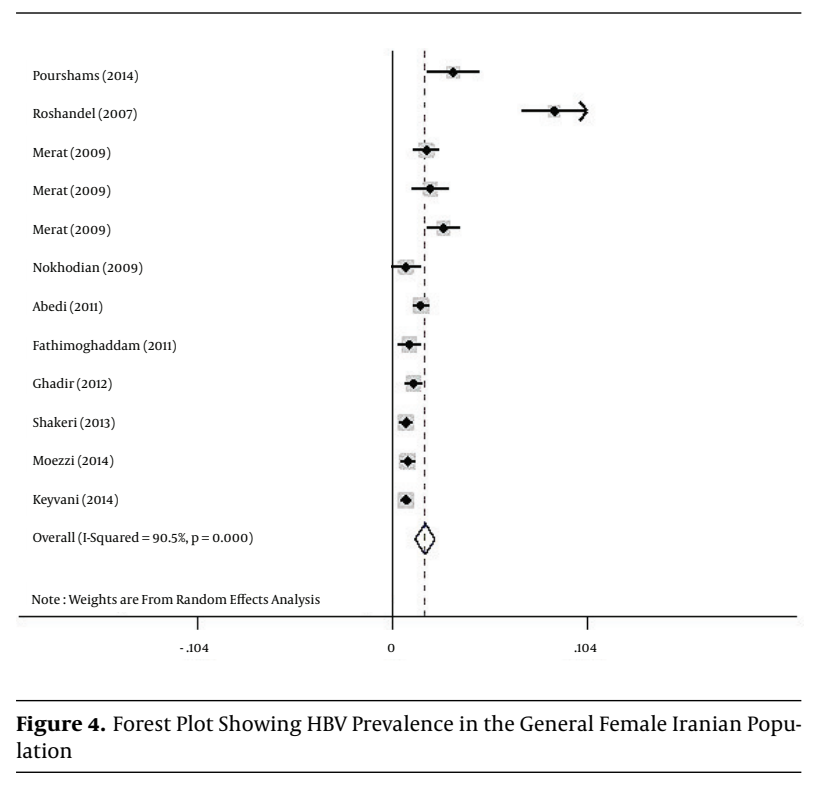

was 2.9\% (95\% CI: 2.5\% - 3.4\%) before 2010 (1993 - 2009) and 1.3\% (95\% CI: 0.9\% - 1.7\%) after 2010 (2010 - 2014) (Figure 5). In the period before 2010, there were two studies with high HBV prevalence of $8.9 \%$ and $5.1 \%(23,25)$. Therefore, we performed sensitivity analysis and estimated HBV prevalence in studies before 2010 with the exclusion of the two mentioned studies. After sensitivity analysis, the prevalence of HBV infection in this period decreased to $2.3 \%$ (95\% CI: $2 \%$ 2.7\%), and overall estimated prevalence of HBV in the general population changed to $1.8 \%$ (95\% CI: $1.6 \%-2.1 \%$ ). After performing cumulative meta-analysis, the prevalence of HBV showed a decrease from 2.5\% in 1993 to $1.7 \%$ in 2014 (Figure 6).

Based on the results of Begg's and Egger's tests for prevalence of HBV infection $(\mathrm{P}=0.005$ and $\mathrm{P}=0.035$, respectively), there was evidence of publication bias in the studies. Therefore, the Trim and Filled method was employed. Using this analysis, HBV prevalence in the general population of Iran was estimated as $2.2 \%$ (95\% CI: 1.9\% $2.6 \%)$.

The results indicated a significant difference within the geographic distribution of HBV infection in the general Iranian population (Table 1, Figure 7). The highest prevalence (6.1\%, 95\% CI: 3.5\% - 8.7\%) was observed in Golestan province (in northeast Iran), followed by Sistan and Baluchestan province (3\%, 95\% CI: $2.3 \%-3.7 \%$ ), while the lowest prevalence was reported from Kermanshah (0.7\%, 95\% CI: $0.4 \%-1.1 \%)$, Kordestan (0.8\%, 95\% CI: 0.4\% - 1.2\%), and Mazandaran (0.9\%, 95\% CI: 0.7\% - 1.2\%).

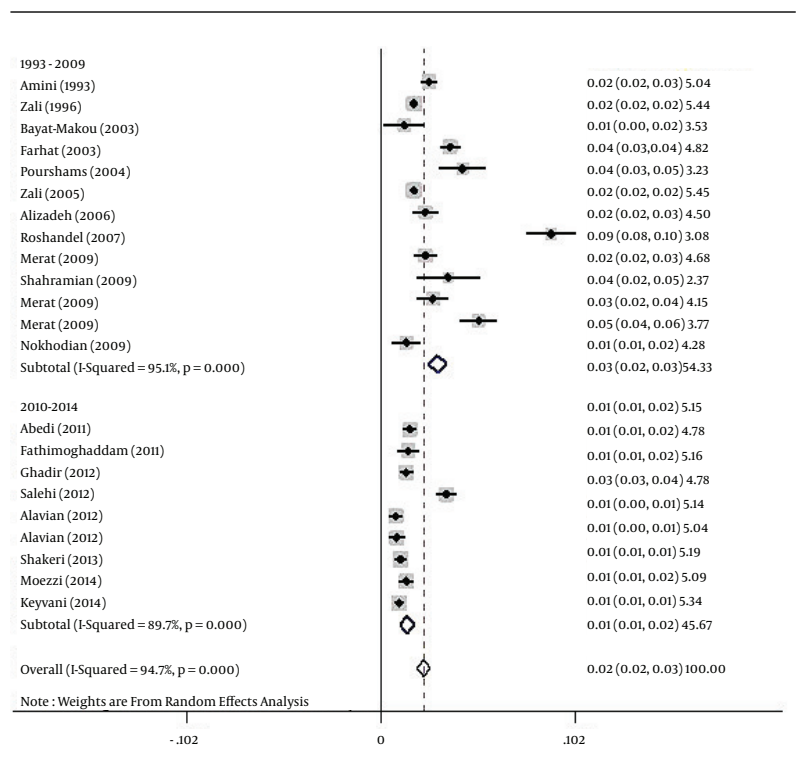

Figure 5. Forest Plot Showing Prevalence of HBV Infection in the General Iranian Population in Two Different Time Periods

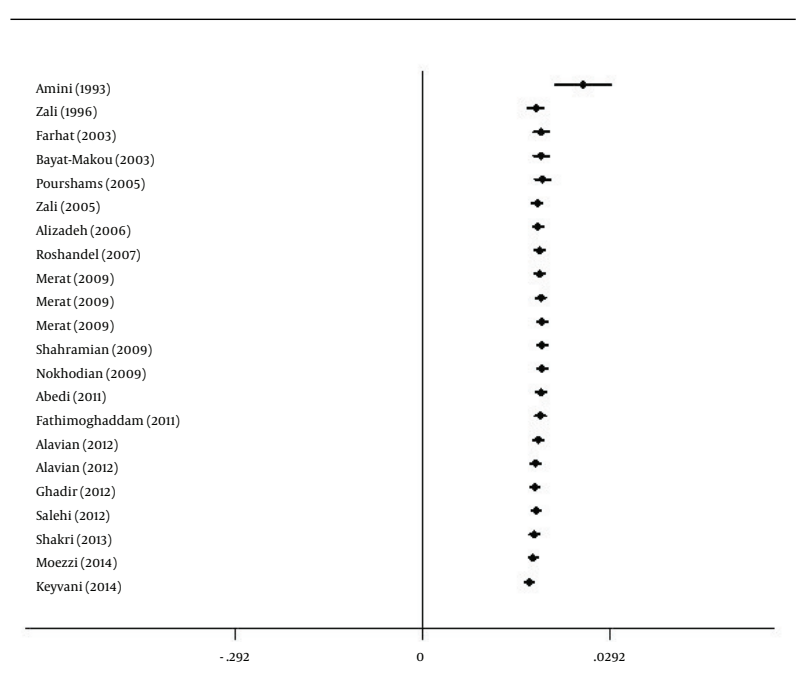

Figure 6. Forest Plot Showing Cumulative Prevalence of HBV Infection in the General Iranian Population According to Time

\section{Discussion}

HBV infection is a major public health problem and considered the leading cause of chronic liver disease in Iran $(1,38)$. In the last decade, HBV prevalence has reduced significantly in Iran (38) because of the infantile mass vaccination program started in 1993, enhancement of people's awareness regarding HBV risk factors, vaccination of highrisk people, and the use of disposable syringes in vaccinations and clinical settings (39-43). 


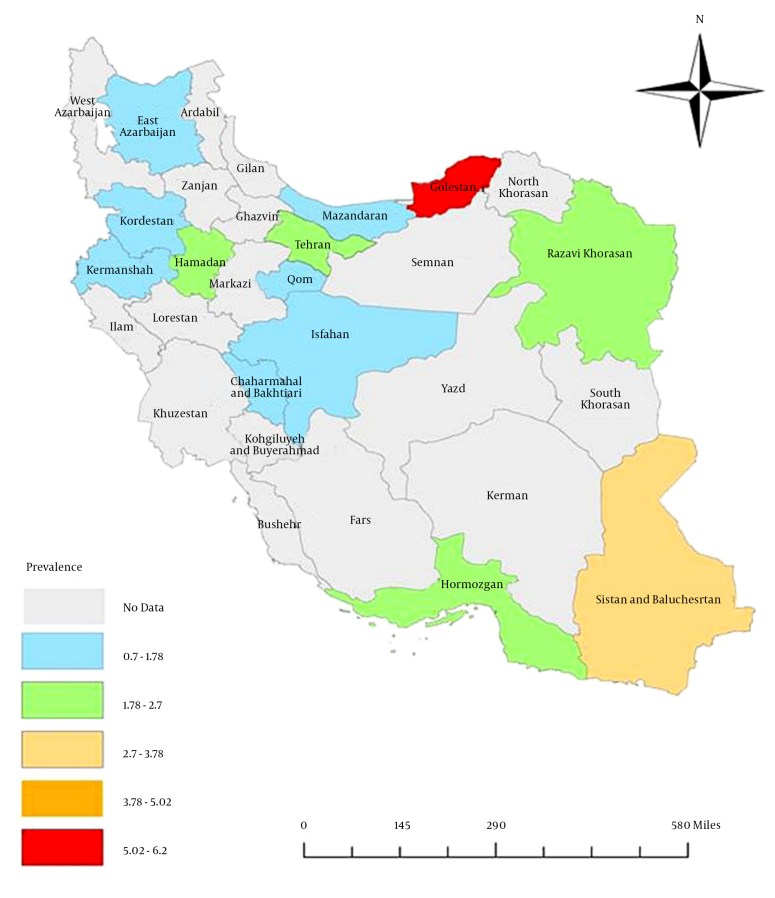

Figure 7. Geographic Distribution of HBV Infection in Iran

Regardless of the present study, two previous systematic reviews have estimated the prevalence of HBV infection in the general population of Iran. Alavian et al. (16) conducted the first systematic review covering 14 studies from 2001 to 2007 . They estimated that HBV prevalence in Iran had a rate of $2.14 \%$ ( $95 \% \mathrm{CI}: 1.9 \%-2.3 \%$ ). In the second systematic review, covering six studies from 1998 to 2005 by Poorolajal et al. (17), the prevalence of HBV in the general Iranian population was estimated at a rate of $2.7 \%$ (95\% CI: $2.2 \%-3.1 \%)$.

In the current study, to obtain an updated estimate of $\mathrm{HBV}$ prevalence in the general Iranian population, a comprehensive review of the literature covering the years 1993 - 2014 was performed. According to the results, the estimated prevalence of $\mathrm{HBV}$ infection in the general population of Iran was $2.2 \%$ (95\% CI: $1.9 \%-2.6 \%$ ), a rate between the previous studies by Alavian et al. (16) and Poorolajal et al. (17).

According to an implementation of preventive measurements, including vaccination programs in last two decades, it is expected that $\mathrm{HBV}$ prevalence will be reduced over time. In the present study, a notable decrease of HBV infection rate was observed within the data published in the period after 2010 (1.3\%) compared to the data published in the period before 2010 (2.9\%). It should be noted that, in the studies before 2010, there are two studies from Golestan province with the HBV prevalence of $8.9 \%$ and $5.1 \%(23,25)$. Following sensitivity analysis and excluding the two studies from Golestan province $(14,23)$, the prevalence of HBV infection in the studies before 2010 and overall prevalence of HBV decreased to $2.3 \%$ (95\% CI: $2 \%$ - 2.7\%) and $1.8 \%$ (95\% CI: $1.6 \%-2.1 \%$ ), respectively.

Despite vaccination having reduced significantly the HBV prevalence and its complications, there are some concerns in this regards, as low efficacy of HBV immunization has been reported by several studies (44-48). Therefore, considering some revisions in HBV vaccination may be more helpful for prevention of HBV infection (49).

In this study, the prevalence of HBV infection in males was higher than females (3\% vs. 1.7\%). This difference might be due to the frequency of more exposure to risk factors, such as occupational risk factors in Iranian males. Also, other risk factors, such as intravenous drug use, multipartnership, and being shaved by common barbers, are more frequent in Iranian men compared to women $(10,14)$.

There was heterogeneity in the geographic distribution of HBV infection and the range of HBV prevalence was extensive, spanning from $0.7 \%$ to $8.9 \%$. This finding suggests that various parts of Iran may differ regarding possible risk factors of HBV infection, and extensive investigations should be performed to determine the geographic distribution of HBV risk factors (16).

It should be noted that only two studies have examined the prevalence of $\mathrm{HBV}$ in the general Iranian population at a national level, and both were conducted before 2,000 (10, 11). Also, at a regional level, little is known about HBV prevalence in the general population in the majority of Iran's provinces. Therefore, implementation of new studies at both the national and regional levels seems to be necessary to achieve real updated information on the HBV prevalence for improving the efficiency of interventions and prevention measurements.

It is worth mentioning that occult HBV infection (patients seronegative for HBsAg but positive for HBV DNA) is very important in public health (50-52), as it has been evidenced that patients with this type of HBV infection are at increased risk of cirrhosis and hepatocellular carcinoma (HCC) $(51,53)$ and also for the risk of HBV transfusion transmission from HbsAg negative blood donors and management of bone marrow and organ transplantations (54, 55). Given that routine detection of HBV infection, which is based on the detection of HbsAg, cannot detect occult HBV infections, implementation of HBV DNA and screening should be considered in epidemiological studies (50).

One of the strengths of the current study is that only studies that really were performed in the general population were included. However, the systematic review by Ala- 
vian et al. (16) included the studies on the general population, blood donors, patients on surgery, and students. Additionally, in the present review, well-designed studies form peer-reviewed journals with proper sampling strategies and reliable laboratory techniques were included. Another strength of this systematic review is that it is truly updated, as it covers studies published up to Jan. 1, 2016.

The present systematic review has some limitations. Iranian national databases are not functional enough because of the insufficiency of search tools, absence of userfriendly advanced search tools, and limited coverage of all national scientific journals, universities research projects, and dissertations. Another major limitation was applying standard keywords for search in Iranian national databases. To solve this problem, all synonyms of standard keywords in both Persian and English languages were subjected to search.

\section{Conclusion}

According to all data on the prevalence of HBV in the general population reviewed in this study, Iran was classified within the low-intermediate HBV prevalence areas ( $2 \%$ - 4.99\%), while according to recent data (after 2010), Iran is classified within the low HBV prevalence areas $(<2 \%)$, indicating that preventive measures conducted in Iran were effective. There is a heterogeneous pattern of distribution of HBV infection between Iran's provinces, indicating the necessity of continuous prevention and control measurements and the implementation of further epidemiologic studies for collecting reliable data on HBV prevalence in different parts of Iran.

\section{Acknowledgments}

The authors would like to thank the staff of the Baqiyatallah research center for gastroenterology and liver diseases.

\section{Footnote}

Funding/Support: This study was supported by the Baqiyatallah research center for gastroenterology and liver diseases.

\section{References}

1. Trepo C, Chan HL, Lok A. Hepatitis B virus infection. Lancet. 2014;384(9959):2053-63. doi: 10.1016/S0140-6736(14)60220-8. [PubMed: 24954675].

2. Chen DS. Toward elimination and eradication of hepatitis B. J Gastroenterol Hepatol. 2010;25(1):19-25. doi: 10.1111/j.14401746.2009.06165.x. [PubMed: 20136972].
3. Ott JJ, Stevens GA, Groeger J, Wiersma ST. Global epidemiology of hepatitis B virus infection: new estimates of age-specific HBsAg seroprevalence and endemicity. Vaccine. 2012;30(12):2212-9. doi: 10.1016/j.vaccine.2011.12.116. [PubMed: 22273662].

4. Chisari FV, Isogawa M, Wieland SF. Pathogenesis of hepatitis B virus infection. Pathol Biol (Paris). 2010;58(4):258-66. doi: 10.1016/j.patbio.2009.11.001. [PubMed: 20116937].

5. Chang ML, Liaw YF. Hepatitis B flares in chronic hepatitis B: pathogenesis, natural course, and management. J Hepatol. 2014;61(6):1407-17. doi: 10.1016/j.jhep.2014.08.033. [PubMed: 25178562].

6. Lozano R, Naghavi M, Foreman K, Lim S, Shibuya K, Aboyans V, et al. Global and regional mortality from 235 causes of death for 20 age groups in 1990 and 2010: a systematic analysis for the Global Burden of Disease Study 2010. Lancet. 2012;380(9859):2095-128. doi: 10.1016/S0140-6736(12)61728-0. [PubMed: 23245604].

7. Andre F. Hepatitis B epidemiology in Asia, the middle East and Africa. Vaccine. 2000;18:S20-2. doi:10.1016/S0264-410X(99)00456-9.

8. Shepard CW, Simard EP, Finelli L, Fiore AE, Bell BP. Hepatitis B virus infection: epidemiology and vaccination. Epidemiol Rev. 2006;28:11225. doi: 10.1093/epirev/mxj009. [PubMed: 16754644].

9. Schweitzer A, Horn J, Mikolajczyk RT, Krause G, Ott JJ. Estimations of worldwide prevalence of chronic hepatitis B virus infection: a systematic review of data published between 1965 and 2013. Lancet. 2015;386(10003):1546-55. doi: 10.1016/S0140-6736(15)61412-X.

10. Zali MR. Epidemiology of hepatitis B in the Islamic Republic of Iran. East Mediterr Health J. 1996;2(2):290-8.

11. Zali MR, Mohammad K, Noorbala AA, Noorimayer B, Shahraz S. Rate of hepatitis B seropositivity following mass vaccination in the Islamic Republic of Iran. East Mediterr Health J. 2005;11(1-2):62-7. [PubMed: 16532672].

12. Alizadeh AH, Ranjbar M, Ansari S, MirArab A, Alavian SM, Mohammad $\mathrm{K}$, et al. Seroprevalence of hepatitis B in Nahavand, Islamic Republic of Iran. East Mediterr Health J. 2006;12(5):528-37. [PubMed: 17333790].

13. Farhat A, Khademi G, Mazlouman SJ. The prevalence of hepatitis B carrier state in Khorassan province of Iran. Saudi Med J. 2003;24(5):54951. [PubMed: 12847639].

14. Gholamreza R, Shahryar S, Abbasali K, Hamidreza J, Abdolvahab M, Khodaberdi K, et al. Seroprevalence of hepatitis B virus and its coinfection with hepatitis D virus and hepatitis $C$ virus in Iranian adult population. Indian J Med Sci. 2007;61(5):263-8. [PubMed: 17478956].

15. You CR, Lee SW, Jang JW, Yoon SK. Update on hepatitis B virus infection. World J Gastroenterol. 2014;20(37):13293-305. doi: 10.3748/wjg.v20.i37.13293. [PubMed: 25309066].

16. Alavian SM, Hajarizadeh B, Ahmadzad-Asl M, Kabir A, BagheriLankarani K. Hepatitis B Virus infection in Iran: A systematic review. Hepat Mon. 2008;8(4):281-94.

17. Poorolajal J, Majdzadeh R. Prevalence of chronic hepatitis B infection in Iran: a review article. J Res Med Sci. 2009;14(4):249-58. [PubMed: 21772891].

18. Moher D, Liberati A, Tetzlaff J, Altman DG. Preferred reporting items for systematic reviews and meta-analyses: the PRISMA statement. Ann Intern Med. 2009;151(4):264-9.

19. Vandenbroucke JP, von Elm E, Altman DG, Gotzsche PC, Mulrow CD, Pocock SJ, et al. Strengthening the Reporting of Observational Studies in Epidemiology (STROBE): explanation and elaboration. PLoS Med. 2007;4(10):e297. doi: 10.1371/journal.pmed.0040297. [PubMed: 17941715].

20. Harris R, Bradburn M, Deeks J, Harbord R, Altman D, Sterne J. Metan: fixed-and random-effects meta-analysis. Stata J. 2008;8(1):3.

21. Harbord RM, Harris RJ, Sterne JA. Updated tests for small-study effects in meta-analyses. Stata J. 2009;9(2):197.

22. Sterne J. A. , Harbord R. M. . Funnel plots in meta-analysis. Stata J. 2004(4):127-41. 
23. Merat S, Rezvan H, Nouraie M, Jamali A, Assari S, Abolghasemi H, et al. The prevalence of hepatitis B surface antigen and anti-hepatitis B core antibody in Iran: a population-based study. Arch Iran Med. 2009;12(3):225-31. [PubMed: 19400598].

24. Pourshams A, Nasiri J, Mohammadkhani A, Nasrollahzadeh D. Hepatitis B in Gonbad-Kavoos: prevalence, risk factors and intrafamilial spreading. Govaresh. 2004;9(4):222-5.

25. Amini S, Mahmoodi MF, Andalibi S, Solati AA. Seroepidemiology of hepatitis B, delta and human immunodeficiency virus infections in Hamadan province, Iran: a population based study. J Trop Med Hyg. 1993;96(5):277-87. [PubMed: 8411302].

26. Fathimoghaddam F, Hedayati-Moghaddam MR, Bidkhori HR, Ahmadi S, Sima HR. The prevalence of hepatitis B antigen-positivity in the general population of Mashhad, Iran. Hepat Mon. 2011;11(5):34650. [PubMed: 22087159].

27. Shakeri MT, Foghanian B, Nomani H, Ghayour-Mobarhan M, Nabavinia MS, Rostami S, et al. The prevalence of hepatitis B virus infection in mashhad, iran: a population-based study. Iran Red Crescent Med J. 2013;15(3):245-8. doi:10.5812/ircmj.8200. [PubMed: 23984006].

28. Shahramian E, Moradi A, Moin AA, noori NM, Solimani G. Hepatitis B sero-markers evaluation in People born. Yafteh. 2009;10(4):107-14.

29. Salehi M, Alavian SM, Tabatabaei SV, Izadi S, Sanei Moghaddam E, Amini Kafi-Abad S, et al. Seroepidemiology of HBV infection in SouthEast of iran; a population based study. Iran Red Crescent Med J. 2012;14(5):283-8. [PubMed: 22829987].

30. Moezzi M, Imani R, Khosravi N, Pourheidar B, Ganji F, Karimi A. Hepatitis B seroprevalence and risk factors in adult population of chaharmahal and bakhtiari province in 2013. Hepat Mon. 2014;14(5):e17398. doi:10.5812/hepatmon.17389. [PubMed: 24910705].

31. Bayat-Makou J, Shahnazi A, Koushavar H. Prevalent infections in north-west region of Tabriz. Med J Tabriz Uni Med Sci. 2003;59:30.

32. Nokhodian Z, Kassaian N, Ataei B, Javadi AA, Shoaei P, Farajzadegan Z, et al. Hepatitis B markers in Isfahan, Central Iran: a population-based study. Hepat Mon. 2009;9(1):12-6.

33. Alavian SM, Tabatabaei SV, Nourizad S, Mansouri F, Khademi N, Kafiabad SA, et al. Seroepidemiology of HBV Infection in KermanshahWest of Iran; a Population Based Study. Jundishapur J Microbio. 2012;5(4):564-9. doi: 10.5812/jjm.4156.

34. Alavian SM, Tabatabaei SV, Ghadimi T, Beedrapour F, Kafi-Abad SA, Gharehbaghian A, et al. Seroprevalence of Hepatitis B Virus Infection and Its Risk Factors in the West of Iran: A Population-based Study. Int JPrev Med. 2012;3(11):770-5. [PubMed: 23189228].

35. Keyvani H, Sohrabi M, Zamani F, Poustchi H, Ashrafi H, Saeedian F, et al. A population based study on hepatitis B virus in northern iran, amol. Hepat Mon. 2014;14(8):e20540. doi: 10.5812/hepatmon.20540. [PubMed: 25237372].

36. Ghadir MR, Belbasi M, Heidari A, Jandagh M, Ahmadi I, Habibinejad H, et al. Distribution and risk factors of hepatitis $B$ virus infection in the general population of Central Iran. Hepat Mon. 2012;12(2):112-7. doi: 10.5812/hepatmon.822. [PubMed: 22509188].

37. Abedi F, Madani H, Asadi A, Nejatizadeh A. Significance of bloodrelated high-risk behaviors and horizontal transmission of hepatitis B virus in Iran. Arch Virol. 2011;156(4):629-35. doi: 10.1007/s00705-0100902-y. [PubMed: 21229276].

38. Alavian SM. Hepatitis B virus infection in Iran; Changing the epidemiology. Arch Clin Infec Dis. 2010;5(1):51-61.

39. Adibi P, Ghassemian R, Alavian SM, Ranjbar M, Mohammadalizadeh $\mathrm{AH}$, Nematizadeh $\mathrm{F}$, et al. Effectiveness of hepatitis B vaccination in children of chronic hepatitis B mothers. Saudi Med J. 2004;25(10):1414-8. [PubMed: 15494813].

40. Kabir A, Alavian SM, Ahanchi N, Malekzadeh R. Combined passive and active immunoprophylaxis for preventing perinatal transmission of the hepatitis B virus in infants born to HBsAg positive mothers in comparison with vaccine alone. Hepatol Res. 2006;36(4):265-71. doi: 10.1016/j.hepres.2006.08.001. [PubMed:16965939].

41. Poorolajal J, Mahmoodi M, Majdzadeh R, Nasseri-Moghaddam S, Haghdoost A, Fotouhi A. Long-term protection provided by hepatitis B vaccine and need for booster dose: a meta-analysis. Vaccine. 2010;28(3):623-31. doi: 10.1016/j.vaccine.2009.10.068. [PubMed: 19887132].

42. Poorolajal J, Mahmoodi M, Majdzadeh R, Nasseri-Moghaddam S, Haghdoost A, Ghalichi L, et al. Seroprotection of hepatitis B vaccine and need for booster dose: a meta-analysis. Hepat Mon 2009;9(4):293-304

43. Poorolajal J, Mahmoodi M, Haghdoost A, Majdzadeh R, NasseriMoghaddam S, Ghalichi L, et al. Booster dose vaccination for preventing hepatitis B. Cochrane Database Syst Rev. 2010(11):CD008256. doi: 10.1002/14651858.CD008256.pub2. [PubMed: 21069704].

44. Al Ghamdi SS, Fallatah HI, Fetyani DM, Al-Mughales JA, Gelaidan AT Long-term efficacy of the hepatitis B vaccine in a high-risk group. J Med Virol. 2013;85(9):1518-22. doi: 10.1002/jmv.23658. [PubMed: 23852676].

45. Elrashidy H, Elbahrawy A, El-Didamony G, Mostafa M, George NM, Elwassief A, et al. Antibody levels against hepatitis B virus after hepatitis B vaccination in Egyptian diabetic children and adolescents. Hum Vaccin Immunother. 2013;9(9):2002-6. doi: 10.4161/hv.25426. [PubMed: 23787761].

46. Masuet-Aumatell C, Ramon-Torrell JM, Casanova-Rituerto A, BanqueNavarro M, Davalos-Gamboa Mdel R, Rodriguez SL. Seroprevalence of hepatitis B in two period birth cohorts of Bolivian children: effect of universal vaccination. Trans R Soc Trop Med Hyg. 2013;107(9):578-83. doi: 10.1093/trstmh/trt057. [PubMed: 23851262].

47. Mizusawa M, Perlman DC, Lucido D, Salomon N. Rapid loss of vaccineacquired hepatitis B surface antibody after three doses of hepatitis B vaccination in HIV-infected persons. Int J STD AIDS. 2014;25(3):201-6. doi: 10.1177/0956462413495820. [PubMed: 24216032].

48. Nisihara R, De Bem RS, Negreiros PH, Utiyama SR, Oliveira NP, Amarante $\mathrm{H}$. Low hepatitis $\mathrm{B}$ vaccine response in children with Down syndrome from Brazil. Child Care Health Dev. 2014;40(4):607-9. doi: 10.1111/cch.12099. [PubMed: 23952481].

49. Rezaee-Zavareh MS, Einollahi B. Hepatitis B vaccination: needs a revision. Hepat Mon. 2014;14(3):e17461. doi: 10.5812/hepatmon.17461. [PubMed: 24734095]

50. Said ZN. An overview of occult hepatitis B virus infection. World J Gas troenterol. 2011;17(15):1927-38. doi: 10.3748/wjg.v17.i15.1927. [PubMed: 21528070].

51. Anvari FA, Alavian SM, Norouzi M, Mahabadi M, Jazayeri SM. Prevalence and molecular analysis of occult hepatitis B virus infection isolated in a sample of cryptogenic cirrhosis patients in iran. Oman Med J. 2014;29(2):92-6. doi: 10.5001/omj.2014.23. [PubMed: 24715933].

52. Keyvani H, Agah S, Kabir A, Alavian SM. Prevalence and risk factors of isolated anti-HBc antibody and occult hepatitis B infection in hemodialysis patients: a nationwide study. Ann Hepatol. 2013;12(2):213-9. [PubMed: 23396732].

53. Pollicino T, Squadrito G, Cerenzia G, Cacciola I, Raffa G, Craxi A, et al Hepatitis B virus maintains its pro-oncogenic properties in the case of occult HBV infection. Gastroenterology. 2004;126(1):102-10. [PubMed: 14699492].

54. Raimondo G, Pollicino T, Romano L, Zanetti AR. A 2010 update on occult hepatitis B infection. Pathol Biol (Paris). 2010;58(4):254-7. doi: 10.1016/j.patbio.2010.02.003. [PubMed: 20303674].

55. Candotti D, Allain JP. Transfusion-transmitted hepatitis B virus infection. J Hepatol. 2009;51(4):798-809. doi: 10.1016/j.jhep.2009.05.020. [PubMed: 19615780]. 